589.

\title{
ON RESIDUATION IN REGARD TO A CUBIC CURVE.
}

[From the Messenger of Mathematics, vol. III. (1874), pp. 62-65.]

THE following investigation of Prof. Sylvester's theory of Residuation may be compared with that given in Salmon's Higher Plane Curves, 2nd Edition (1873), pp. 133-137 :

If the intersections of a cubic curve $U_{3}$ with any other curve $V_{\dot{n}}$ are divided in any manner into two systems of points, then each of these systems is said to be the residue of the other; and, in like manner, if starting with a given system of points on a cubic curve we draw through them a curve of any order $V_{n}$, then the remaining intersections of this curve with the cubic constitute a residue of the original system of points.

If the number of points in the original system is $=3 p$, then the number of points in the residual system is $=3 q$; and if we again take the residue, and so on indefinitely, the number of points in each residue will be $\equiv 0$ (Mod. 3); viz. we can never in this way arrive at a single point. But if the number of points in the original system be $3 p \pm 1$, then that in the residual system will be $3 q \mp 1$; and we may in an infinity of different ways arrive at a residue consisting of a single point; or say at a "residual point," viz. after an odd number of steps if the original number of points is $=3 p-1$, but after an even number of steps if the original number of points is $=3 p+1$. But starting from a given system of points on a given cubic curve, the residual point, however it is arrived at, will be one and the same point; this is Prof. Sylvester's theorem of the residuation of a cubic curve. For instance, starting with two given points on the cubic curve, the line joining these meets the curve in a third point, which is the residual point; any other process leading to a residual point must lead to the same point. Thus if through the 2 points we draw a conic, meeting the cubic besides in 4 points; through these a conic meeting the cubic besides 
in 2 points; and through this a line meeting the cubic besides in 1 point; this will be the before-mentioned residual point.

The general proof is such as in the following example:

Take on the cubic $U_{3}$ a system of $3 \kappa-2$ points, say the points $\alpha$ : through these a curve $V_{k}$, besides meeting the cubic in $3 k-3 \kappa+2$ points $\beta$ : and through these a curve $P_{k-\kappa+1}$, besides meeting the cubic in a point $C$. And again through the $3 \kappa-2$ points $\alpha$ a curve $W_{k^{\prime}}$, besides meeting the cubic in $3 k^{\prime}-3 \kappa+2$ points $\beta^{\prime}$ : and through these a curve $Q_{k^{\prime}-\kappa+1}$, besides meeting the cubic in a single point; this will be the point $C$.

The proof consists in showing that we have a curve $A_{k+k^{\prime}-\kappa-2}$ such that

$$
A_{k+k^{\prime}-\kappa-2} U_{3}=Q_{k^{\prime}-\kappa+1} V_{k}+P_{k-\kappa+1} W_{k^{\prime}} \text {. }
$$

For this observe that

$Q_{k^{\prime}-\kappa+1}$ meets $W_{k^{\prime}}$ in $3 k^{\prime}-3 \kappa+2$ points $\beta^{\prime}$ and besides in $k^{\prime 2}-k^{\prime}(\kappa+2)+3 \kappa-2$ points $\epsilon^{\prime}$;

$P_{k-\kappa+1}$ meets $V_{k}$ in $3 k-3 \kappa+2$ points $\beta$ and besides in $k^{\prime 2}-k(\kappa+2)+3 \kappa-2$ points $\epsilon$;

$P_{k-\kappa+1}, Q_{k^{\prime}-\kappa+1}$ meet in $(k-\kappa+1)\left(k^{\prime}-\kappa+1\right)$ points $C$;

$V_{k}, W_{k^{\prime}}$ meet in $3 \kappa-2$ points $\alpha$ and $k k^{\prime}-3 \kappa+2$ points $a$;

$Q_{k^{\prime}-\kappa+1} V_{k}$ and $P_{k-\kappa+1} W_{k^{\prime}}$ meet in

$$
\begin{aligned}
& k k^{\prime}-k(\kappa-1)-k^{\prime}(\kappa-1)+(\kappa-1)^{2} \text { points } C \\
& 3 k^{\prime} \quad-3 \kappa+2 \quad, \quad \beta^{\prime} \\
& k^{\prime 2} \quad-k^{\prime}(\kappa+2)+3 \kappa-2 \quad \text { " } \epsilon^{\prime} \\
& 3 k \quad-3 \kappa+2 \quad, \quad \beta \\
& k^{2}-k(\kappa+2) \quad+3 \kappa-2 \quad " \quad \epsilon \\
& k k^{\prime}-3 \kappa+2 \quad " \quad a \\
& \frac{3 \kappa-2}{\left(k+k^{\prime}\right)^{2}-(2 \kappa-2)\left(k+k^{\prime}\right)+(\kappa-1)^{2}} \quad \Rightarrow \quad \alpha \\
& =\left(k+k^{\prime}-\kappa+1\right)^{2} \text { points. }
\end{aligned}
$$

Every $\left(k+k^{\prime}-\kappa+1\right)$ thic through

$$
\frac{1}{2}\left(k+k^{\prime}-\kappa+1\right)\left(k+k^{\prime}-\kappa+4\right)-1
$$

of these points passes through all.

Now $A_{k+k^{\prime}-\kappa-2}$ may be drawn to pass through

$$
\frac{1}{2}\left(k+k^{\prime}-\kappa-2\right)\left(k+k^{\prime}-\kappa+1\right)
$$

of the points $a$. 
Hence $A_{k+k^{\prime}-\kappa-2} U_{3}$ is a $\left(k+k^{\prime}-\kappa+1\right)$ thic through

$$
\begin{aligned}
& \frac{1}{2}\left(k+k^{\prime}-\kappa-2\right)\left(k+k^{\prime}-\kappa+1\right) \\
& =\frac{1}{2}\left(k+k^{\prime}\right)^{2}-\frac{1}{2}(2 \kappa+1)\left(k+k^{\prime}\right)+\frac{1}{2}\left(\kappa^{2}+\kappa-2\right) \text { points } a \\
& 3 \kappa-2 \quad, \quad \alpha \\
& 3 k \quad-3 \kappa+2 \quad, \quad \beta \\
& 3 k^{\prime} \quad-3 \kappa+2 \quad, \quad \beta^{\prime} \\
& \frac{1}{2}\left(k+k^{\prime}\right)^{2}+\left(-\kappa+\frac{5}{2}\right)\left(k+k^{\prime}\right)+\frac{1}{2} \kappa^{2}-\frac{5}{2} \kappa+1 \\
& =\frac{1}{2}\left\{\left(k+k^{\prime}\right)^{2}+\left(k+k^{\prime}\right)(-2 \kappa+5)+(\kappa-1)(\kappa+4)-2\right\} \\
& =\frac{1}{2}\left(k+k^{\prime}-\kappa+1\right)\left(k+k^{\prime}-\kappa+4\right)-1
\end{aligned}
$$

of the points in question; and therefore through all. Whence

$$
A_{k+k^{\prime}-\kappa-2} U_{3}=Q_{k^{\prime}-\kappa+1} V_{k}+P_{k-\kappa+1} W_{k^{\prime}}
$$
are

Also $U_{3}$ meets $Q_{k^{\prime}-\kappa+1} V_{k}$ in $3\left(k+k^{\prime}-\kappa+1\right)$ of the $\left(k+k^{\prime}-\kappa+1\right)^{2}$ points, viz. these

$$
\begin{array}{rcc}
3 \kappa-2 & \text { points } & \alpha, \\
3 k-3 \kappa+2 & " & \beta \\
3 k^{\prime}-3 \kappa+2 & " & \beta^{\prime} \\
1 & " & C
\end{array}
$$

and $A_{k+k^{\prime}-\kappa-2}$ meets $Q_{k^{\prime}-\kappa+1} V_{k}$ in $\left(k+k^{\prime}-\kappa-2\right)\left(k+k^{\prime}-\kappa+1\right)$,

that is, in

of the

$$
\left(k+k^{\prime}\right)^{2}+\left(k+k^{\prime}\right)(-2 \kappa-1)+\kappa^{2}+\kappa-2
$$

viz. these are

$$
\left(k+k^{\prime}-\kappa+1\right)^{2} \text { points, }
$$

$$
\begin{array}{crrrr}
k k^{\prime} & +\left(k+k^{\prime}\right)(-\kappa+1)+\kappa^{2}-2 \kappa & \text { points } & C \\
k^{\prime 2} & -k^{\prime}(\kappa+2) & +3 \kappa-2 & \prime & \epsilon^{\prime} \\
k^{2} & -k(\kappa+2) & +3 \kappa-2 & \Rightarrow & \epsilon \\
k k^{\prime} & -3 \kappa+2 & \Rightarrow & a \\
\hline\left(k+k^{\prime}\right)^{2}+\left(k+k^{\prime}\right)(-2 \kappa-1)+\kappa^{2}+\kappa-2 & \text { points. }
\end{array}
$$

Hence $U_{3}$ passes through 1 of the points $C$, that is, through an intersection of $Q_{k^{\prime}-\kappa+1}$ and $P_{k-\kappa+1}$, that is, $Q_{k^{\prime}-\kappa+1}$ and $P_{k-\kappa+1}$ intersect $U_{3}$ in a common point $C$; which was the theorem to be proved.

In the particular case $3 \kappa-2=10, k=k^{\prime}=4$, the theorem is, given on a cubic 10 points, if through these we draw a quartic meeting the cubic besides in 2 points; 
and through these a line meeting the cubic besides in a point $C$; then this is a fixed point, independent of the particular quartic. And the proof is as follows: we have

$U$ a cubic through 10 points $\alpha$;

$V$ a quartic through the 10 points, and besides meeting the cubic in 2 points $\beta$;

$W$ a quartic through the 10 points, and besides meeting the cubic in 2 points $\beta^{\prime}$;

$P$ the line joining the two points $\beta$, and besides meeting $V$ in two points $\epsilon$;

$Q$ the line joining the two points $\beta^{\prime}$, and besides meeting $W$ in two points $\epsilon^{\prime}$; $P, Q$ meet in the point $C$;

$U, V$ meet in the 10 points $\alpha$, and besides in 6 points $a$;

$A$ a conic through 5 of the points $a$.

Then quintics $Q V, P W$ meet in the 10 points $\alpha, 2$ points $\beta, 2$ points $\epsilon, 2$ points $\beta^{\prime}$, 2 points $\epsilon^{\prime}, 6$ points $a$ and 1 point $C$. Every quintic through 19 of these passes through the 25. But we have $A U$, a quintic through 5 points $a$, and the 10 points $\alpha$, 2 points $\beta$ and 2 points $\beta^{\prime}$; hence $A U$ passes through all the remaining points, or we have

$$
A U=Q V+P W
$$

$P$ passes through $\beta, \beta, \epsilon, \epsilon, C$,

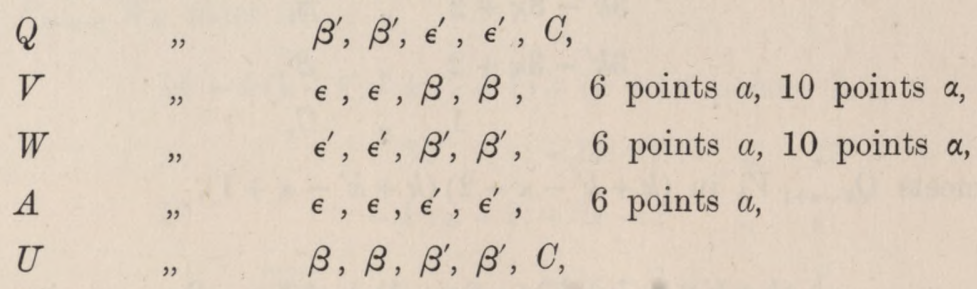

or, what is the same thing,

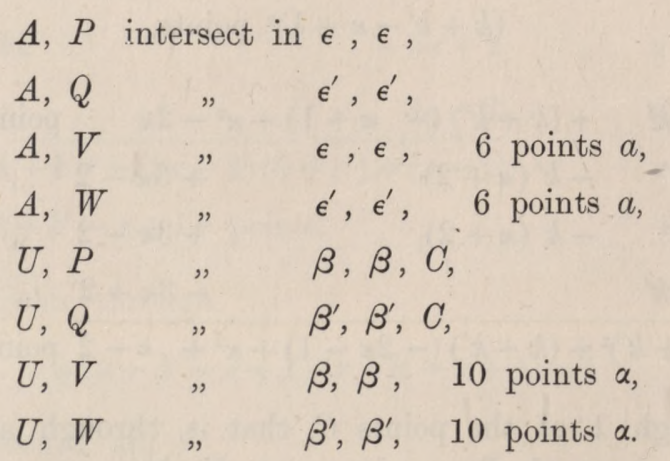

In particular $U, P, Q$ intersect in the point $C$; that is, $C$ as given by the intersection of $U$ by the line $P$; and as given by the intersection of $U$ by the line $Q$; is one and the same point. 